\title{
Loneliness: contemporary insights into causes, correlates, and consequences
}

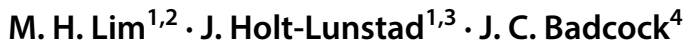

Published online: 11 June 2020

○) Springer-Verlag GmbH Germany, part of Springer Nature 2020

Loneliness is not a new phenomenon but in recent years, there has been a growing interest in understanding how feelings of 'perceived social isolation' can influence our health and wellbeing. Objective indicators of social isolation-such as living alone and number of social connections-have well-demonstrated links with poorer health outcomes [1]. However, the latest evidence indicates that feeling lonely is also associated with a multitude of poorer health outcomes, ranging from an increased risk of depression and dementia [2], increased risk of heart disease and stroke [3] and higher levels of inflammatory responses [4] to name a few. Indeed, those who are socially isolated (odds ratio $=1.29 ; 95 \% \mathrm{CI}$ $1.06,1.56$ ), living alone (odds ratio $=1.32 ; 95 \%$ CI 1.14 , 1.53 ), or those who are lonely (odds ratio $=1.26,95 \% \mathrm{CI}$ $1.04,1.53)$ are at increased risk of earlier mortality [5].

Current "hotspots" in loneliness research include studies examining how perceived social isolation influences mental health symptoms [6] and disorders [7-9], older [10] and younger adults [11], workplace productivity [12, 13], and social media use [14]. The contributions to this special issue illustrate some of the progress, possibilities, and problems in contemporary research on loneliness, including two systematic reviews [15, 16], one conceptual review [17], two pilot studies evaluating a novel approach to reduce loneliness in young people with psychosis $[18,19]$, and two studies exploring personalized approaches to reduce loneliness [20, 21].

First, Ma et al. (2019) provide a review of the effectiveness of interventions targeting subjective and objective

\section{H. Lim \\ mlim@swin.edu.au}

1 Iverson Health Innovation Research Institute, Swinburne University of Technology, Melbourne, VIC, Australia

2 Centre for Mental Health, Swinburne University of Technology, Melbourne, VIC, Australia

3 Brigham Young University, Provo, UT 84602, USA

4 University of Western Australia, Perth, WA, Australia social isolation in people with different mental health problems [15]. The authors examined: (1) interventions that alter maladaptive cognitions about others (e.g., cognitive-behavioural therapy); (2) social skills training and psychoeducation programmes (e.g., family psychoeducation therapy); (3) supported socialisation (e.g., peer support groups); and (4) community approaches (e.g., social prescribing). When considering only those studies specifically targeting interventions for loneliness, Ma et al. [15] found that the most promising results emerged for cognition modification interventions.

The study by Mihalopoulos et al. [16] highlights the importance of understanding the economic burden of loneliness and/or social isolation and is one of the first to evaluate the cost-effectiveness of interventions targeting loneliness and/or social isolation. The authors reported that all but one of the published cost-of-illness studies indicated greater healthcare costs for individuals experiencing loneliness and/or social isolation. However, Mihalopoulos et al. [16] noted that these costs "are likely to be underestimated" due to the limited evidence available, particularly for younger populations. Of the interventions included in this systematic review, the authors highlight "promising" cost-effective interventions that involved increasing social and peer-contact.

In the conceptual review by Lim, Eres, and Vasan [17], the authors outline emerging and established correlates and risk factors associated with loneliness. Importantly, the review identified two newer variables of interest in loneliness research, namely workplaces and the use of digital communication. Lim and colleagues also highlight the complexity of loneliness and introduce a new conceptual model that describes how multiple risk factors/correlates can affect loneliness severity. The authors stress that there is no 'onesize-fits-all' solution for loneliness; rather, how loneliness is resolved is dependent on an individual's circumstances and available resources. 
The next two studies illustrate how a theory-driven approach (i.e., strengths-based positive psychology) was used to develop an intervention targeting loneliness. In a brief report, Lim et al. [18] evaluate the feasibility and acceptability of a positive psychology intervention group program called Positive Connect for young people experiencing psychosis. This 6-week positive psychology group intervention was designed to help young people identify their strengths and practice interpersonal skills that could be used to build close relationships with others. Preliminary evidence presented suggests that the program is both feasible and acceptable for this patient population. Encouragingly, exploratory analyses also indicate a positive benefit for reducing loneliness over time.

In the second related study, the authors describe the development of the same positive psychology program being translated and delivered via a digital smartphone app called + Connect [19]. The authors used focus groups to steer the design, functionality, and language of the 6-week program, to facilitate consumer engagement. Using an innovative blend of content, concepts were conveyed via text and videos (featuring young people with lived experience, academics, and actors). The feasibility, acceptability, and usability of + Connect is reported for a pilot sample $(N=12)$ of young people with psychosis, along with tentative evidence of a benefit in reducing loneliness.

The next study by Tymoszuk et al. [20] looked at the impact of arts engagement on loneliness, specifically, whether the frequency of receptive arts engagement was associated with lower odds of loneliness in older adults. The authors used existing data drawn from the English Longitudinal Study of Ageing (ELSA), analyzing participants (over 50 years old) with complete data on engagement with arts, covariate variables, and loneliness from the second wave $(n=6222)$ for cross-sectional analyses, and loneliness data from the seventh wave $(n=3127)$ for longitudinal analyses. In cross-sectional findings, frequent engagement with arts activities was associated with lowers odds of loneliness. However, longitudinal findings were less supportive, including no evidence that cinema engagement reduced loneliness.

Finally, consistent with the need to develop individualized solutions, Wang et al. [21] examined variables associated with loneliness for individuals (18-75 years old) leaving a Crisis Resolution Team (CRT). A total of 399 participants, with most reporting depression/anxiety disorders (35.0\%), followed by schizophrenia/psychosis (27.0\%), bipolar affective/manic (16.3\%), and other disorders (8.4\%) were included in the analyses. Results showed that loneliness was more severe for individuals who have more mental health contact over the years (2-10 years), compared to those who have less than 3 months, and those who have less than 1 year of mental health service contact. Higher loneliness was also associated with more severe affective, positive or negative symptoms. Those who had depression, anxiety, personality disorders or other disorders compared with those who had psychotic disorders were also lonelier. In those with a mental disorder, lower loneliness was also associated with greater social network size and increased neighbourhood social capital [21].

Many of the studies in this special issue, draw attention to the importance of the need for rigorous loneliness research so that we can extend our knowledge on how loneliness impacts on health. Accordingly, it is crucial that we measure loneliness as a key variable of interest alongside specific health-related outcomes in future research. In doing so, we are also more likely to measure loneliness in a comprehensive way using psychometrically validated assessment tools, avoiding dichotomous measurement of loneliness to draw accurate comparisons across different samples. Given the significant public health implications, the current studies also call attention to the need for a routine and consistent approach to assessing and documenting loneliness as psychosocial "vital signs" of care [7].

Many of the reviewed studies also highlight the need to conduct longitudinal research to clarify the relationships between loneliness and poorer health outcomes. Pertinent questions such as 'are people with pre-existing health problems more predisposed to feeling lonely', or 'are people who are lonely more predisposed to developing problematic health conditions?' have significant, real-world implications for the development of effective treatments.

In addition, it is also clear that greater attention is needed in the development and evaluation of solutions/interventions for loneliness. Designing consumer relevant programs can improve uptake and adherence to programs and Lim et al.'s study shows an example of how consumers are increasingly engaged within research to help tailor evidence-based material to be more engaging to relevant groups [19]. However, there is currently mixed evidence of what is helpful and unhelpful for loneliness in terms of solutions. Hence, solely relying on consumers' ideas concerning what they can do to address their own loneliness may be helpful for engagement but may not be necessarily effective. What is more crucial is the rigorous evaluation of theory-driven evidencebased interventions that are intended to reduce loneliness, and researchers need to move swiftly from pilot evaluations to high quality, adequately powered randomised controlled trials (RCTs). Furthermore, economic evaluations of interventions should be more frequently included in RCTs, to further augment the evidence-base. This involves including both resource use, cost and utility information, and ensuring that the economic evaluation examines both the costs and benefits in healthcare.

Clearly, there are many unanswered questions. For example, if loneliness is a common experience, when does loneliness make a significant negative impact on health outcomes? 
When does loneliness as a transient experience become a chronic issue? Much work is required to understand the negative impact of loneliness on ourselves, our community and the society we live in and we look forward to learning more about loneliness within a dynamic social world.

\section{Compliance with ethical standards}

Conflicts of interest On behalf of all authors, the corresponding author states that there is no conflict of interest.

\section{References}

1. Pantell M, Rehkopf D, Jutte D et al (2013) Social isolation: a predictor of mortality comparable to traditional clinical risk factors. Am J Public Health 11:2056-2062. https://doi.org/10.2105/ AJPH.2013.301261

2. Meltzer H, Bebbington P, Dennis MS et al (2013) Feelings of loneliness among adults with mental disorder. Soc Psychiatry Psychiatr Epidemiol 1:5-13. https://doi.org/10.1007/s0012 7-012-0515-8

3. Hawkley LC, Burleson MH, Berntson GG, Cacioppo JT (2003) Loneliness in everyday life: cardiovascular activity, psychosocial context, and health behaviors. J Pers Soc Psychol 1:105. https:// doi.org/10.1037/0022-3514.85.1.105

4. Steptoe A, Owen N, Kunz-Ebrecht SR, Brydon L (2004) Loneliness and neuroendocrine, cardiovascular, and inflammatory stress responses in middle-aged men and women. Psychoneuroendocrinology 5:593-611. https://doi.org/10.1016/s0306-4530(03)00086 $-6$

5. Holt-Lunstad J, Smith TB, Layton JB (2010) Social relationships and mortality risk: a meta-analytic review. PLoS Med 7:e1000316. https://doi.org/10.1371/journal.pmed.1000316

6. Lim MH, Rodebaugh TL, Zyphur MJ, Gleeson JF (2016) Loneliness over time: the crucial role of social anxiety. J Abnorm Psychol 5:620-630. https://doi.org/10.1037/abn0000162

7. Badcock JC, Mackinnon A, Waterreus A et al (2018) Loneliness in psychotic illness and its association with cardiometabolic disorders. Schizophr Res 204:90-95. https://doi.org/10.1016/j.schre s.2018.09.021

8. Badcock JC, Shah S, Mackinnon A et al (2015) Loneliness in psychotic disorders and its association with cognitive function and symptom profile. Schizophr Res 1-3:268-273. https://doi. org/10.1016/j.schres.2015.10.027

9. Lim MH, Gleeson JFM, Alvarez-Jimenez M, Penn DL (2018) Loneliness in psychosis: a systematic review. Soc Psychiatry
Psychiatr Epidemiol 3:221-238. https://doi.org/10.1007/s0012 7-018-1482-5

10. Cacioppo JT, Hawkley LC, Thisted RA (2010) Perceived social isolation makes me sad: 5-year cross-lagged analyses of loneliness and depressive symptomatology in the Chicago health, aging, and social relations study. Psychol Aging 2:453-463. https://doi. org/10.1037/a0017216

11. Lim MH, Rodebaugh TL, Eres R et al (2019) A pilot digital intervention targeting loneliness in youth mental health. Front Psychiatry 10:604. https://doi.org/10.3389/fpsyt.2019.00604

12. Ozcelik H (2011) Barsade S (2011) Work loneliness and employee performance. Acad Manag Proc 1:1-6. https://doi.org/10.5465/ ambpp.2011.65869714

13. Ozcelik H, Barsade SG (2018) No employee island: workplace loneliness and job performance. Acad Manag J 6:2343-2366. https://doi.org/10.5465/amj.2015.1066

14. Nowland R, Necka EA, Cacioppo JT (2017) Loneliness and social internet use: pathways to reconnection in a digital world? Perspect Psychol Sci 1:70-87. https://doi.org/10.1177/1745691617713052

15. Ma R, Mann F, Wang J et al (2019) The effectiveness of interventions for reducing subjective and objective social isolation among people with mental health problems: a systematic review. Soc Psychiatry Psychiatr Epidemiol. https://doi.org/10.1007/s0012 7-019-01800-z

16. Mihalopoulos C, Le LK, Chatterton ML et al (2019) The economic costs of loneliness: a review of cost-of-illness and economic evaluation studies. Soc Psychiatry Psychiatr Epidemiol. https://doi.org/10.1007/s00127-019-01733-7

17. Lim MH, Eres R, Vasan S (2020) Loneliness in the 21st century: an update on correlates, risk factors and potential solutions. Soc Psychiatry Psychiatr Epidemiol. https://doi.org/10.1007/s0012 7-020-01889-7

18. Lim MH, Penn DL, Thomas N, Gleeson JFM (2019) Is loneliness a feasible treatment target in psychosis? Soc Psychiatry Psychiatr Epidemiol. https://doi.org/10.1007/s00127-019-01731-9

19. Lim MH, Gleeson JFM, Rodebaugh TL, Eres R, Long KM, Casey K, Abbott J-AM, Thomas N, Penn DL (2019) A pilot digital intervention targeting loneliness in young people with psychosis. Soc Psychiatry Psychiatr Epidemiol. https://doi.org/10.1007/s0012 7-019-01681-2

20. Tymoszuk U, Perkins R, Fancourt D, Williamon A (2019) Crosssectional and longitudinal associations between receptive arts engagement and loneliness among older adults. Soc Psychiatry Psychiatr Epidemiol. https://doi.org/10.1007/s00127-019-01764 $-0$

21. Wang J, Lloyd-Evans B, Marston L et al (2019) Epidemiology of loneliness in a cohort of UK mental health community crisis service users. Soc Psychiatry Psychiatr Epidemiol. https://doi. org/10.1007/s00127-019-01734-6 